\section{An immunohistochemical study of matrix proteins in the craniofacial cartilage in midterm human fetuses}

\author{
S. Shibata, ${ }^{1}$ Y. Sakamoto, ${ }^{2}$ O. Baba, ${ }^{3}$ \\ C. Qin, ${ }^{4}$ G. Murakami, ${ }^{5}$ B.H. $\mathrm{Cho}^{6}$ \\ 'Maxillofacial Anatomy, Department of \\ Maxillofacial Biology, Graduate School, \\ Tokyo Medical and Dental University, \\ Japan \\ ${ }^{2}$ Basic Sciences of Oral Health Care, \\ Graduate School, Tokyo Medical and \\ Dental University, Japan \\ ${ }^{3}$ Oral Function and Molecular Biology, \\ Ohu University School of Dentistry, \\ Koriyama, Japan \\ ${ }^{4}$ Department of Biomedical Sciences, \\ Baylor College of Dentistry, Texas A \& M \\ University, System Health Science Center, \\ Dallas, TX, USA \\ ${ }^{5}$ Division of Internal Medicine, Iwamizawa \\ Koujin-kai Hospital, Japan \\ ${ }^{6}$ Department of Surgery, Faculty of \\ Medicine, Chonbuk National University, \\ Jeonju, South Korea
}

\section{Abstract}

Immunohistochemical localization of collagen types I, II, and X, aggrecan, versican, dentin matrix protein (DMP)-1, martix extracellular phosphoprotein (MEPE) were performed for Meckel's cartilage, cranial base cartilage, and mandibular condylar cartilage in human midterm fetuses; staining patterns within the condylar cartilage were compared to those within other cartilaginous structures. Mandibular condylar cartilage contained aggrecan; it also had more type I collagen and a thicker hypertrophic cell layer than the other two types of cartilage; these three characteristics are similar to those of the secondary cartilage of rodents. MEPE immunoreactivity was first evident in the cartilage matrix of all types of cartilage in the human fetuses and in Meckel's cartilage of mice and rats. MEPE immunoreactivity was enhanced in the deep layer of the hypertrophic cell layer and in the cartilaginous core of the bone trabeculae in the primary spongiosa. These results indicated that MEPE is a component of cartilage matrix and may be involved in cartilage mineralization. DMP-1 immunoreactivity first became evident in human bone lacunae walls and canaliculi; this pattern of expression was comparable to the pattern seen in rodents. In addition, chondroid bone was evident in the mandibular (glenoid) fossa of the temporal bone, and it had aggrecan, collagen types I and $\mathrm{X}, \mathrm{MEPE}$, and DMP-1 immunoreactivity; these findings indicated that chondroid bone in this region has phenotypic expression indicative of both hypertrophic chondrocytes and osteocytes.

\section{Introduction}

Mandibular condylar cartilage is a component of temporomandibular joint (TMJ) as well as a growth cartilage in craniofacial region. ${ }^{1,2}$ In addition, this cartilage has traditionally been classified as secondary cartilage in embryology. ${ }^{3,4}$ The term secondary cartilage is not an official anatomical term, but widely accepted in the research field of craniofacial development. Secondary cartilage has several definitions, one of the widest being that it appears later in embryonic development, untouched by the primary cartilaginous skeleton. A narrower definition is that it arises from the periostea of membrane bone after (secondary to) bone formation. Our findings from histological and histochemical studies of mice support this narrow definition., ${ }^{5,6}$ Mandibular angular/coronoid/symphyseal cartilage and os penis cartilage in rodents are classified as secondary cartilage, ${ }^{3,4,7}$ while Meckel's cartilage and cranial base cartilage belong to primary cartilage. ${ }^{7}$ Mandibular condylar cartilage differs somewhat from primary cartilage in, for example, its time of appearance, its cell alignment, the invasion pattern of capillaries, and the distribution of growth factors. ${ }^{3,8,9}$

Meanwhile, many kinds of collagenous and non-collagenous extracellular matrix proteins are used as marker molecules of cartilage and bone. Type I collagen is generally used as a marker for fibrous connective tissue, bone, and dentin; ${ }^{7,10-13}$ aggrecan and type II collagen are used as makers for mature cartilage., ${ }^{5,10-14}$ In addition, type $\mathrm{X}$ collagen is expressed in the hypertrophic cell zone of growth cartilage. $5,7,13$ Versican is a large, non-cartilaginous proteoglycan that is expressed in precartilaginous mesenchymal condensations, dental pulp, brain, and various other mesenchymal tissues. ${ }^{14-17}$ In addition to these molecules, many members of the small integrin-binding ligand N-linked glycoproteins (SIBLING) family have been identified recently. Among them, dentin matrix protein-1 (DMP-1) was initially reported as an odontoblast-specific molecule, ${ }^{18}$ but it was later found to be strongly expressed in osteocytes $^{19-22}$ and weakly expressed in other mineralized tissues such as cartilage, enamel, and dental pulp..$^{22,23}$ Matrix extracellular phosphoprotein (MEPE) is another SIBLING family protein that is also more strongly expressed in
Correspondence: Shunichi Shibata, Maxillofacial Anatomy, Department of Maxillofacial Biology, Graduate School, Tokyo Medical and Dental University, 1-5-45, Yushima, Bunkyo-ku, Tokyo 113-5349, Japan.

E-mail: sshibata.mfa@tmd.ac.jp

Key words: condylar cartilage, human fetus, extracellular matrix, MEPE, DMP-1.

Acknowledgments: this work was supported by Grant-in-Aid for Scientific Research (No. 22592044) from Ministry of Education, Culture, Sports, Science, and Technology of Japan. We thank Mr. Toshimitsu Yamamoto for his invaluable technical assistance in tissue preparation.

Contributions: SS, experimental system design experiments performing, data collecting, manuscript writing; YS, OB, experiments performing, data collecting; $\mathrm{CQ}, \mathrm{MG}$, data discussion, manuscript review and revision; $\mathrm{BHC}$, experimental system design, samples collecting, experiments performing, data discussion.

Received for publication: 31 August 2013. Accepted for publication: 14 October 2013.

This work is licensed under a Creative Commons Attribution NonCommercial 3.0 License (CC BYNC 3.0).

(C) Copyright S. Shibata et al., 2013

Licensee PAGEPress, Italy

European Journal of Histochemistry 2013; 57:e39 doi:10.4081/ejh.2013.e39

osteocytes than in osteoblasts; ${ }^{24-26}$ MEPE, like DMP-1, is also expressed in odontoblasts/odontoblastic cells. ${ }^{27-30}$ MEPE expression in cartilage reportedly occurs only in late-stage hypertrophic chondrocytes in $c$-Src -deficient mice ${ }^{31}$ and during regeneration of fractured calluses in mice, ${ }^{32}$ but MEPE is not normally expressed in cartilage matrix. Furthermore, proliferating cell nuclear antigen (PCNA) is expressed in the nuclei of cell during the DNA synthesis phase of the cell cycle, and widely used to identify the proliferating cell zone of growth cartilage.$^{33}$ Immunohistochemical/in situ hybridization studies of extracellular matrix have mainly been conducted with rodent cartilage and bone including craniofacial cartilage.

In human fetuses, detailed immunohistochemical studies have been performed in cartilage other than craniofacial region. ${ }^{34,35}$ Smith et al..$^{34}$ made detailed immunohistochemical studies of human fetal limb bud cartilage, and they demonstrated that type II collagen and aggrecan are present throughout the entire cartilage matrix and that aggrecan immunoreactivity is also present in ligaments and tendons. Smith et al. ${ }^{35}$ also conducted an immunohistochemical analysis of versican expression 
in spinal tissues of human fetuses; they demonstrated that versican immunolocalization is evident along with fibrillar components in the annular lamellae of the outer annulus fibrosus. However, immunohistochemical studies of these molecules have not been performed for any type of human fetal craniofacial cartilage, including Meckel's cartilage, cranial base cartilage, or mandibular condylar cartilage.

In human fetuses, structural features of the TMJ, including condylar cartilage, are well studied ${ }^{36-41}$ moreover, immunohistochemical analyses have been performed on the articular disc. ${ }^{42-44}$ However, characteristics of condylar (secondary) cartilage that have been identified and documented in rodents have not been assessed in human fetuses.

Thus, the first aim of this study is to perform immunohistochemistry of extracellular matrices for the craniofacial cartilage of midterm human fetuses, and to compare expression patterns of these molecules in secondary cartilage (condylar cartilage) with that in primary cartilage (Meckel's cartilage or cranial base cartilage). Furthermore, immunohistochemical studies of DMP-1 and MEPE have never been performed in human fetal tissue. Thus, the another aim of this study is to perform immunohistochemistry of these molecules to confirm whether the expression patterns in human fetal craniofacial cartilage/bone are similar to those in rodents.

\section{Materials and Methods}

This study was performed in accordance with the provisions of the Declaration of Helsinki 1995 (as revised in Edinburgh). The use of fetuses for research was approved by the Chonbuk National University (Korea) Ethics Committee. All specimens were normal based on macroscopic observations. The entire group comprised nine mid-term fetuses: two fetuses at 10 weeks of gestation, one fetus at 13 weeks, three fetuses at 15 weeks, and three fetuses at 16 weeks were examined. Each foetus was immersed in $4 \%$ paraformaldehyde $(0.1 \mathrm{M}$ phosphate buffer, $\mathrm{pH}$ 7.4) for $7 \mathrm{~d}$ at room temperature. The specimens were decalcified with ethylenediaminetetra-acetic acid, dehydrated with graded series of ethanol, and embedded in paraffin. Sagittal or frontal sections (6- $\mu \mathrm{m}$ thick) were cut serially at the head region. For general histologic observations, toluidine blue staining was performed with some sections; immunohistochemical analyses of matrix protein expression were performed with other sections. In addition, Institute of Cancer Research (ICR) mice and Sprague-Dawley (SD) rats were obtained from Sankyo
Laboratories (Tokyo, Japan), and maintained under standard conditions at the Animal Center of Tokyo Medical and Dental University. The animal-use protocol conformed to the National Institutes of Health guidebook and was reviewed and approved by the Screening Committee for Animal Research of the Tokyo Medical and Dental University (No. 0130081A). Three fetal mice from embryonic day (E)14.0 and three fetal rats from E16.0 were used in the present study. Following ether anesthesia, pregnant mice or rats were killed by cervical dislocation, and the heads of the fetal animals were removed and fixed with $4 \%$ paraformaldehyde; frontally cut paraffin sections were prepared as described above.

\section{Antibodies used and immunohisto- chemistry}

Mouse monoclonal anti-aggrecan G1 domain (12/21/1C6: diluted 1:25) and anti-versican G1 domain (12C5:diluted 1:25) antibod- ies were obtained from the Developmental Studies Hybridoma Bank. Rabbit polyclonal anti-versican GAG- $\alpha$ domain (diluted 1:20) was from Merck Millipore (Chemicon; Temecula, CA, USA). Rabbit polyclonal anticollagen types I (diluted 1:200), II (diluted 1:100), and X (diluted 1:200) antibodies were from LSL (Tokyo, Japan). Each of these antibodies has been used in previous immunohistochemical studies. ${ }^{5,11,15,31}$ Mouse monoclonal anti-PCNA (proliferating cell nuclear antigen) antibody (diluted 1:50) was from Thermo Fisher Scientific (Fremont, CA, USA). The rabbit polyclonal antibody against MEPE, LF-155 (diluted 1:200), was kindly supplied by Dr. Larry W. Fisher (Matrix Biology Unit, Craniofacial and Skeletal Disease Branch, NIDCR, NIH, Bethesda, MD, USA). The mouse monoclonal antibody against a $57-\mathrm{kDa}$ fragment of rat DMP-1 (diluted 1:500) has been created and well characterized. ${ }^{20}$ Each of these antibodies has also been used in a previous immunohistochemical study. ${ }^{20,31}$
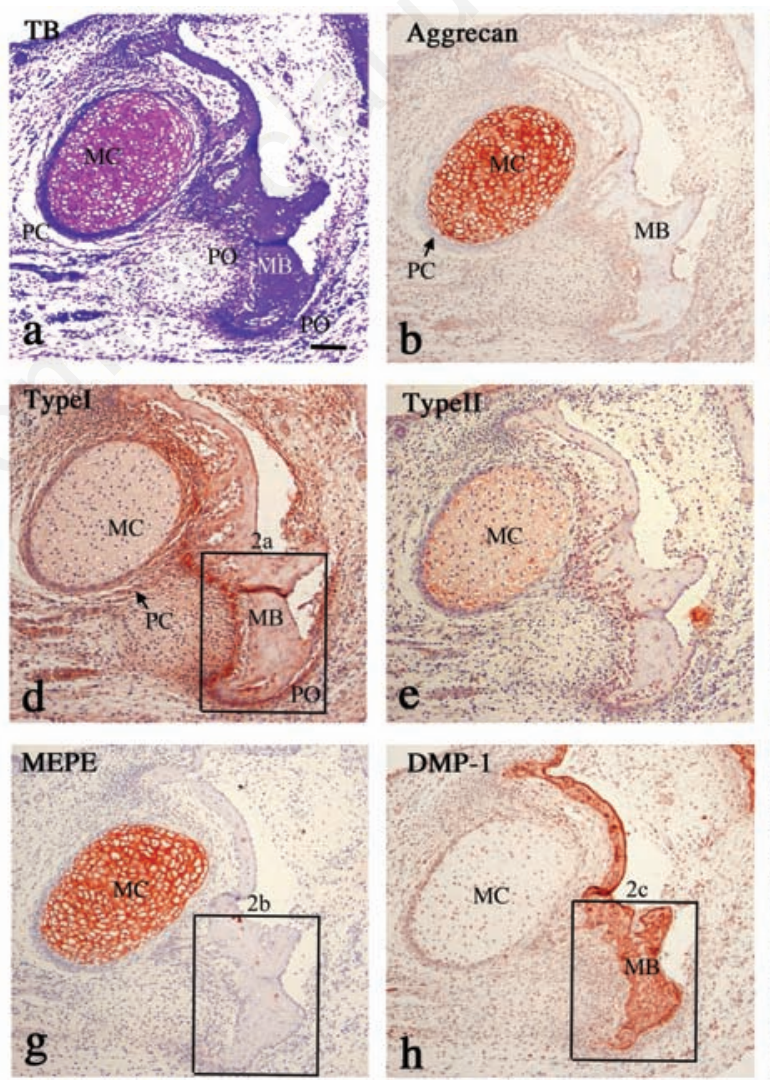
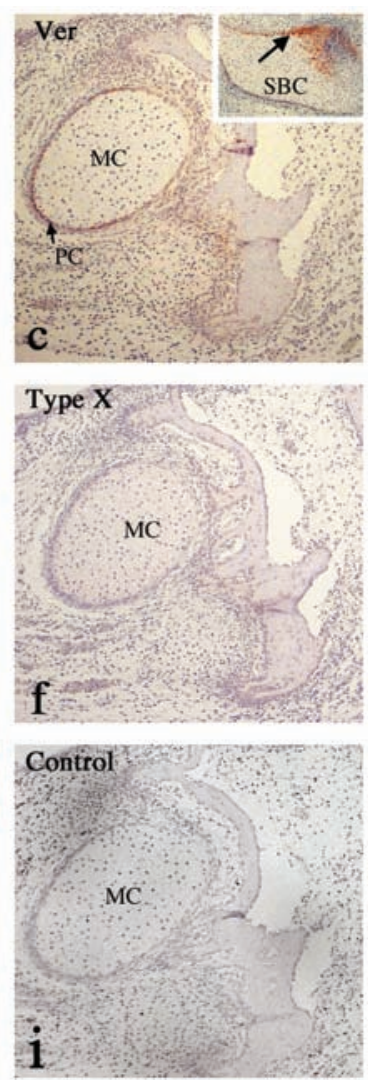

Figure 1. Frontal sections cut at mandibular body region of ossifying mandible at 10 weeks of gestation. Toluidine blue staining (a) and immunohistochemistry for aggrecan (b), versican (c), collagen types I (d), II (e), and X (f), MEPE (g) and DMP-1 (h). A negative control reacted with normal rabbit IgG (i). Meckel's cartilage showed immunoreactivity for aggrecan (b), type II collagen (e), and MEPE (g). Perichondrium (PC) showed immunoreactivity for versican (c), and type I collagen (d). Mandibular bone showed strong DMP-1 immunoreactivity (h). MC, Meckel's cartilage; SBC, sphenoid bone cartilage; PC, perichondrium; MB, mandibular bone. Scale bars: $100 \mu \mathrm{m}$. 
Before antibody addition, all epitopes (except for those on PCNA) were exposed by treating the respective tissue section with testicular hyaluronidase $(25 \mathrm{mg} / \mathrm{mL}$ in phosphatebuffered saline, $30 \mathrm{~min}, 37^{\circ} \mathrm{C}$; Sigma Chemical Co., St. Louis, M0, USA). Sections prepared for aggrecan immunohistochemistry were reduced and alkylated as previously described. ${ }^{15,31}$ PCNA epitopes were retrieved by incubating the sections in $10 \mathrm{mM}$ citrate buffer at $100^{\circ} \mathrm{C}$ for $5 \mathrm{~min}$. The Histofine $\mathrm{SAB}$ kit (Nichirei, Tokyo, Japan) was used to perform streptavidin-biotin labeling as previously described. ${ }^{5,15,16,31}$ A MOM kit (Vector laboratories, Burlingame, CA, USA) was used for DMP1 immunostaining of mouse sections and to block endogenous mouse IgG activity. The sections were treated with 3-amino-9-ethylcarbazole (AEC, red) or diaminobenzidine (DAB, brown) to visualize protein localization. Negative control sections were incubated with normal rabbit or mouse IgG $(10 \mu \mathrm{g} / \mathrm{mL})$ rather than with primary antibodies. Sections were observed after counterstaining with hematoxylin and observed by a light microscope (Provis, Olympus, Tokyo, Japan). Images were captured by a CCD camera (DP12, Olympus, Tokyo, Japan). We evaluated the localization of immunoreactivity in extracellular matrix in the present study except for PCNA (nuclear localization). Although we did not accept some quantitative analyzes, we examined two different samples at 10 weeks of gestation and six different samples at 15/16 weeks of gestation to confirm the consistency of results obtained.

\section{Results \\ Meckel's cartilage and mandibular bone at 10 weeks of gestation}

In human fetuses at 10 weeks of gestation, Meckel's cartilage was clearly evident at the medial side of the ossifying mandibular body. Compact bone had already formed in this region (Figure 1a). Strong aggrecan immunoreactivity was evident in Meckel's cartilage, but not in the bone or perichondrium associated with Meckel's cartilage (Figure 1b). Weak versican immunoreactivity was evident in the perichondrium of Meckel's cartilage and the sphenoid bone cartilage in the same section (Figure 1c). Strong type I collagen immunoreactivity was evident in the periosteum of the mandibular bone and the perichondrium of Meckel's cartilage, but type I collagen immunoreactivity was weak in the bone matrix (Figure 1d). Type II collagen immunoreactivity was exclusively detected in Meckel's cartilage (Figure 1e). No type X collagen immunoreactivity was detected in tissue sections from the 10-week specimens (Figure 1f). Strong MEPE immunoreactivity was evident in Meckel's cartilage and in a few bone lacunae, but not in any other tissue examined (Figure 1g). Strong DMP-1 immunoreactivity was evident in the bone matrix, but not in any other tissue examined (Figure 1h). Negative control sections showed no positive immunoreactivity (Figure 1i). Immunoreactivity within compact bone in this region was examined under higher magnification. Immunoreactivity of type I collagen described above was further confirmed (Figure 2a). Notably, at this magnification, MEPE immunoreactivity was evident in a few bone lacunae walls (arrows in Figure 2 b,d). DMP-1 immunoreactivity was evident in most of bone lacunae walls and canaliculi (arrows and asterisk in Figure 2 c,e). MEPE immunoreactivity was also evident in Meckel's cartilage in fetal mice at E14.0 and in fetal rats at E16.0 (Figure
$2 \mathrm{f}, \mathrm{g})$. DMP-1 immunoreactivity was evident in the mandibular bone of fetal mice and fetal rats, but not in the Meckel's cartilage of either animal (Figure $2 \mathrm{~h}, \mathrm{i}$ ).

\section{Condylar cartilage at 16 weeks of gestation}

In the human fetuses at 15 weeks and at 16 weeks of gestation, the mandibular condylar cartilage had formed in addition to Meckel's cartilage. The findings from the two stages (total 6 species) were similar; therefore, the findings from 16 weeks of gestation will be described in detail as representative of both stages.

All images in Figures 3, 4, and 5 were from obliquely cut sagittal sections taken from a fetus at 16 weeks of gestation. Each type of cartilage was clearly identifiable based on tissue metachromasia following staining with tolui-
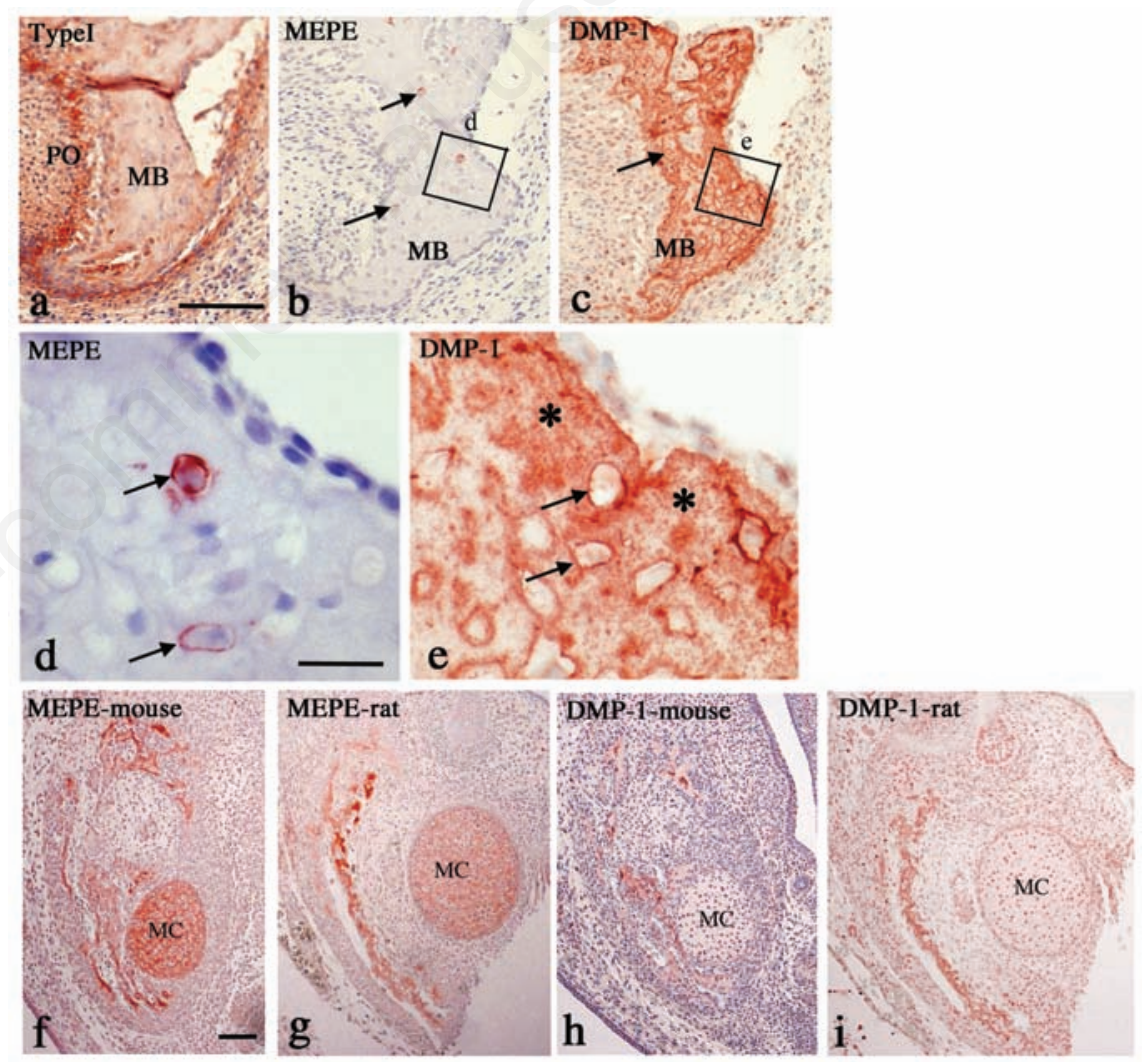

Figure 2. a,b,c) are higher magnifications of ossifying mandibular body indicated by rectangular areas in Figure 1d, g, h, respectively. d,e) are higher magnifications of rectangular areas in b and c, respectively. Frontally cut Meckel's cartilage in mice at E14.0 (f, h) and rats at E16.0 (g,i). Immunostaining for type I collagen (a), MEPE (b,d,f,g) and DMP-1 (c,e,h,i). Strong type I collagen immunoreactivity was evident in the periosteum (a). MEPE immunoreactivity was evident in a few bone lacunae walls (arrows in d). DMP-1 immunoreactivity was evident in most of bone lacunae walls (arrows in e) and canaliculi (asterisks in e). Meckel's cartilage in mice and rats showed MEPE immunoreactivity (f,g), but not DMP-1 immunoreactivity $(h, i)$. PO, periosteum; MB, mandibular bone; MC, Meckel's cartilage. Scale bars: a-c, f-i) $100 \mu \mathrm{m}$; d,e) $20 \mu \mathrm{m}$. 
dine blue. Meckel's cartilage was transversally cut at the upper part (Figure 3a) and at the lower part (Figure $3 \mathrm{~b}$ ) of the condylar process. When sections of condylar cartilage were cut obliquely from the upper part, vascular canals were evident as described previously. ${ }^{36}$ The mandibular fossa was evident superior to the condylar cartilage (Figure 3a). Endochondral ossification had started in the lower part of the condylar cartilage, and primary spongiosa had formed inferior to the condylar cartilage (Figure 3b). Meckel's cartilage at this stage showed similar expression patterns of immunohistochemistry for each molecule (data not shown, except for versican and type II immunostaining in Figure 3 e2,g2).

Images of immunoreactivity against matrix proteins in the upper and lower parts of condylar cartilage are presented in parallel in Figures 3 and 4, and corresponding images of the mandibular fossa and future joint cavity are presented in Figure 5. Mérida-Velasco et $a l .{ }^{37}$ described the fetal mandibular condyle as comprising five layers: the articular, mesenchymal, chondroblastic, chondrocyte, and hypertrophic cell layers. We adopted this classification for the present study (Figure 3 c1,c2). Bone tissue (bone collar) had formed around the condylar cartilage in the middle and lower parts of the condylar process. In addition, capillaries had invaded opened lacunae in the primary spongiosa (Figure $3 \mathrm{c} 2$ ).

Aggrecan immunoreactivity was evident from the chondroblastic layer to the hypertrophic cell layer (arrows in Figure $3 \mathrm{~d} 1, \mathrm{~d} 2$ ), but not in the articular layer and mesenchymal layer (arrowheads in Figure 3d1). Versican immunoreactivity was evident in the future upper and lower joint cavity (arrow in Figure $3 \mathrm{e}$, also see Figure $5 \mathrm{c}$ ), and in the perichondrium of Meckel's cartilage (arrow in Figure $3 \mathrm{e} 2$ ), but was not evident in the condylar cartilage (Figure 3 e1,e2). Type I collagen immunoreactivity was evident from the articular layer to the chondroblastic layer (arrows in Figure 3f1), in the bone collar (including the periosteum), and in the deepest layer of the hypertrophic cell layer (arrows in Figure 3f2). Type II collagen immunoreactivity was evident from the chondroblastic layer to the hypertrophic cell layer (arrows in Figure $3 \mathrm{~g} 1, \mathrm{~g} 2$ ), but the staining in these layers was typically less intense than that in Meckel's cartilage in the same sections. Type X collagen immunoreactivity was evident from the chondrocyte layer to the hypertrophic cell layer (arrows in Figure $3 \mathrm{~h} 1, \mathrm{~h} 2)$. MEPE immunoreactivity was evident from the chondroblastic layer to the hypertrophic cell layer (arrows in Figure 4 a1,a2), and staining was most intense in the deep layer of the hypertrophic cell layer (large arrow in Figure 4a2). DMP-1 immunoreactivity was intense in the bone collar (Figure 4 b1,b2) and weak in the deep layer of the hypertrophic cell layer (arrows in Figure 4b2). PCNA immunoreactivity was evident in a large number of cells in the mesenchymal layer (arrows in Figure 4c1) and in a small number of cells in the chondroblastic layer (arrowheads in Figure $4 \mathrm{c} 1)$, but it was rare in the chondrocyte and
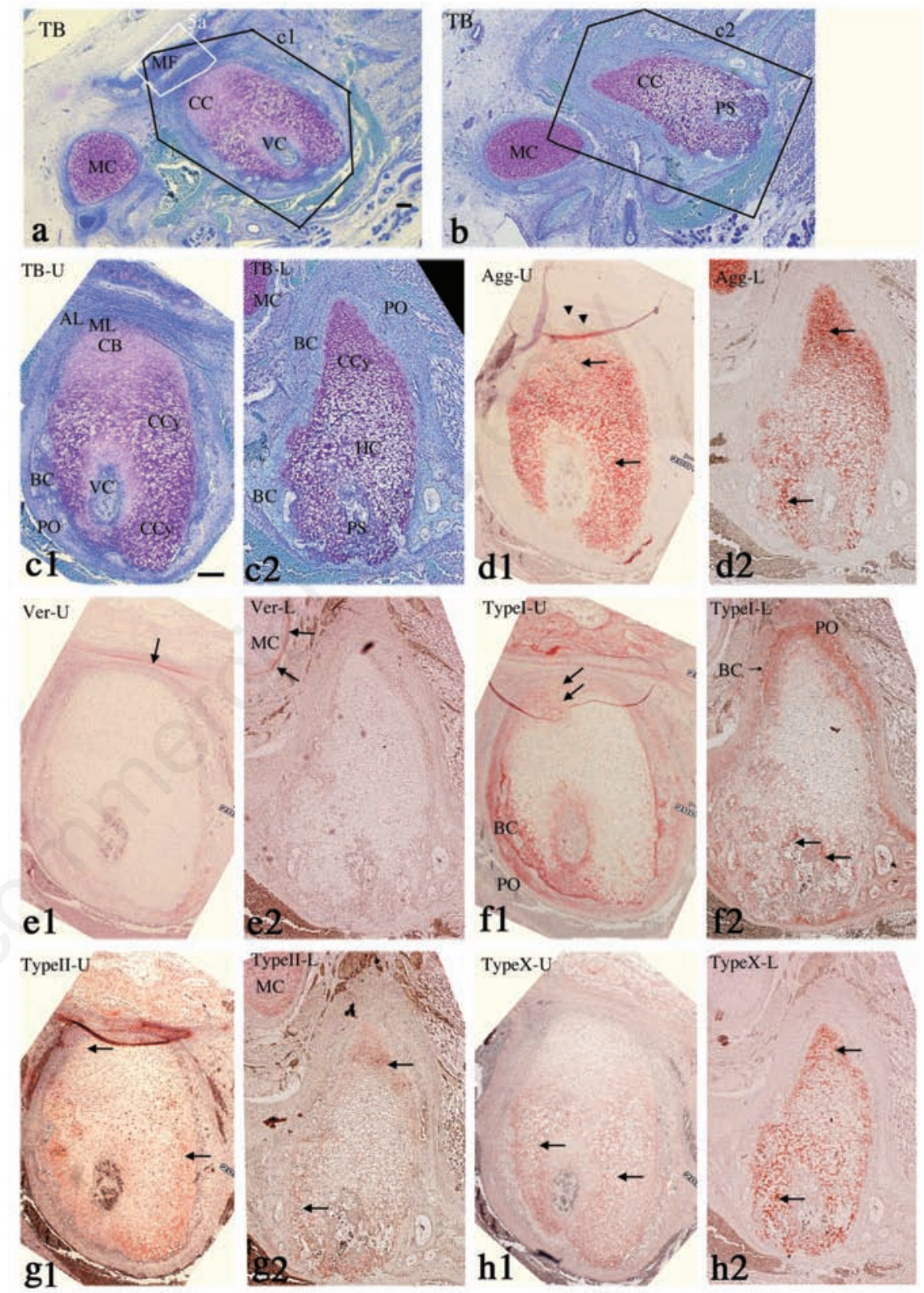

Figure 3. Obliquely-cut sagittal sections at the upper part (a) and lower part (b) of the condylar process stained with toluidine blue at 16 weeks of gestation. $c 1, c 2)$ are higher magnification of rectangular areas of a and $b$, respectively. $d 1-h, d 2-h 2$ ) are adjacent sections to $\mathrm{c1}$ and $\mathrm{c2}$, respectively, and immunostaining for aggrecan (d1,d2), versican (e1,e2), collagen types I (f1,f2), II (g1, g2), and X (h1,h2). Condylar cartilage showed immunoreactivity for aggrecan and type II collagen mainly in the lower layers $(\mathrm{d} 1, \mathrm{~d} 2, \mathrm{~g} 1, \mathrm{~g} 2)$, and for type $I$ collagen in the upper layers $(\mathrm{f} 1, \mathrm{f} 2)$, and for type $X$ collagen exclusively in the hypertrophic cell layer $(\mathrm{h} 1, \mathrm{~h} 2)$. Versican immunoreactivity was evident in the future joint cavity and in the perichondrium of Meckel's cartilage (arrows in e1,e2). CC, condylar cartilage; MC, Meckel's cartilage; MF, mandibular fossa; VC, vascular canal; PS, primary spongioza; BC, bone collar; PO, periosteum; AL, articular layer; $\mathrm{ML}$, mesenchymal layer; $\mathrm{CB}$, chondroblast layer; $\mathrm{CCy}$, chondrocyte layer; $\mathrm{HC}$, hypertrophic cell layer. Scale bars: $200 \mu \mathrm{m}$. 


\section{Mandibular (glenoid) fossa and anlagen of articular cavity at 16 weeks of gestation}

Many lacunae of various sizes within the ossifying mandibular fossa were evident in higher magnification images, and anlagen of the future upper cavity and the joint cavity were identifiable as fissures between the condylar cartilage and the mandibular fossa. An area between these anlagen was apparently the anlagen of the future articular disc (Figure 5a). Representative images of the immunoreactivity of matrix proteins are presented in Figure 5 b-h. Most lacunae showed immunoreactivity for type I collagen (arrows in Figure 5d) and DMP-1 (arrows in Figure 5h), and a small number of lacunae showed immunoreactivity for aggrecan (arrows in Figure 5b), type $X$ collagen (arrow in Figure 5f), and MEPE (arrows in Figure 5g); however, no lacunae showed versican (arrows in Figure $5 c$ ) or type II collagen (arrows in Figure 5e) immunoreactivity. The anlagen of the future articular joint cavity showed intense versican immunoreactivity (arrowheads in Figure 5c) and slight type I collagen immunoreactivity (arrowheads in Figure 5d). Negative control sections showed no positive immunoreactivity (Figure 5i).

\section{Cranial base cartilage at 16 weeks of gestation}

The cranial base cartilage underwent endochondral ossification at 16 weeks of gestation. This cartilage was subdivided into three zones - the proliferative, maturation, and hypertrophic cell zones - as is the case for long bone cartilage, ${ }^{1}$ primary spongiosa was evident in the diaphysis. Bone collar had formed around the diaphysis, and the periosteum of this bone collar was continuous with perichondrium around the metaphysis (Figure 6a).

Representative images of matrix protein immunoreactivity are shown in Figure 6 b-h. Aggrecan immunoreactivity (arrows in Figure $6 \mathrm{~b}$ ) and type II collagen (arrows in Figure 6e) immunoreactivity were evident throughout the entire cartilage matrix, but versican immunoreactivity was not evident in any region examined (arrow in Figure 6c). Type I collagen immunoreactivity was evident in the bone collar, the periosteum, and the perichondrium (Figure 6d). Type X collagen immunoreactivity was detected exclusively in the hypertrophic cell zone and cartilaginous core of the bone trabeculae in primary spongiosa (arrows in Figure 6f). MEPE immunoreactivity was evident throughout the entire cartilage matrix (arrows in Figure 6g), and it was enhanced in the cartilaginous core (large arrow in Figure $6 \mathrm{~g})$. Diffuse DMP-1 immunoreactivity was evident in the bone collar and bone trabeculae in the primary spongiosa (large arrow in Figure $6 \mathrm{~h})$ as well as in the hypertrophic cell zone (arrow in Figure 6h). PCNA immunoreactivity was detected in a large number of cells in the perichondrium (arrows in Figure 6i), but it was rarely detected in the cartilage, which included the proliferative zone (Figure 6i). Negative control sections showed no positive immunoreactivity (Figure 6j).

\section{Discussion}

\section{Expression of collagen types, aggrecan and proliferating cell nuclear antigen in primary and secondary cartilage}

Both Meckel's cartilage and cranial base cartilage are classified as primary cartilage. ${ }^{3,7}$ Aggrecan and type II collagen are generally accepted as molecular markers of mature cartilage matrix, and both molecules were evident throughout the entire matrix.

Smith et al..$^{34}$ performed detailed immunohistochemical studies of limb bud cartilage in human fetuses and demonstrated that aggre- can and type II collagen are present throughout the cartilage matrix; notably, aggrecan immunoreactivity was also seen in ligaments and tendons. We did not see aggrecan immunoreactivity in any tissue other than cartilage. This slight discrepancy between our findings and those of Smith et al. ${ }^{34}$ may be due to differences in the antibodies used because the antibody used in our study exclusively recognizes cartilage matrix. ${ }^{15,31}$ Type $\mathrm{X}$ collagen is a marker of hypertrophic chondrocytes in growth cartilage, and this molecule was evident in the hypertrophic cell zone of cranial base cartilage, and it was not evident in Meckel's cartilage. Thus, our findings in human fetal cartilage were consistent with previously reported distribution patterns of type X collagen in other animals. Type I collagen is a marker for fibrous connective tissue, bone, and dentin; this molecule was evident in the perichondrium, the periosteum, and bone collar of the cranial base cartilage; these findings indicated that generally accepted distribution patterns of type I collagen in other animals are comparable to those in the human fetus. Smith et al. ${ }^{35}$ used anti-type I collagen antibody in an immunohistochemical study of the spine of human fetuses and demonstrated
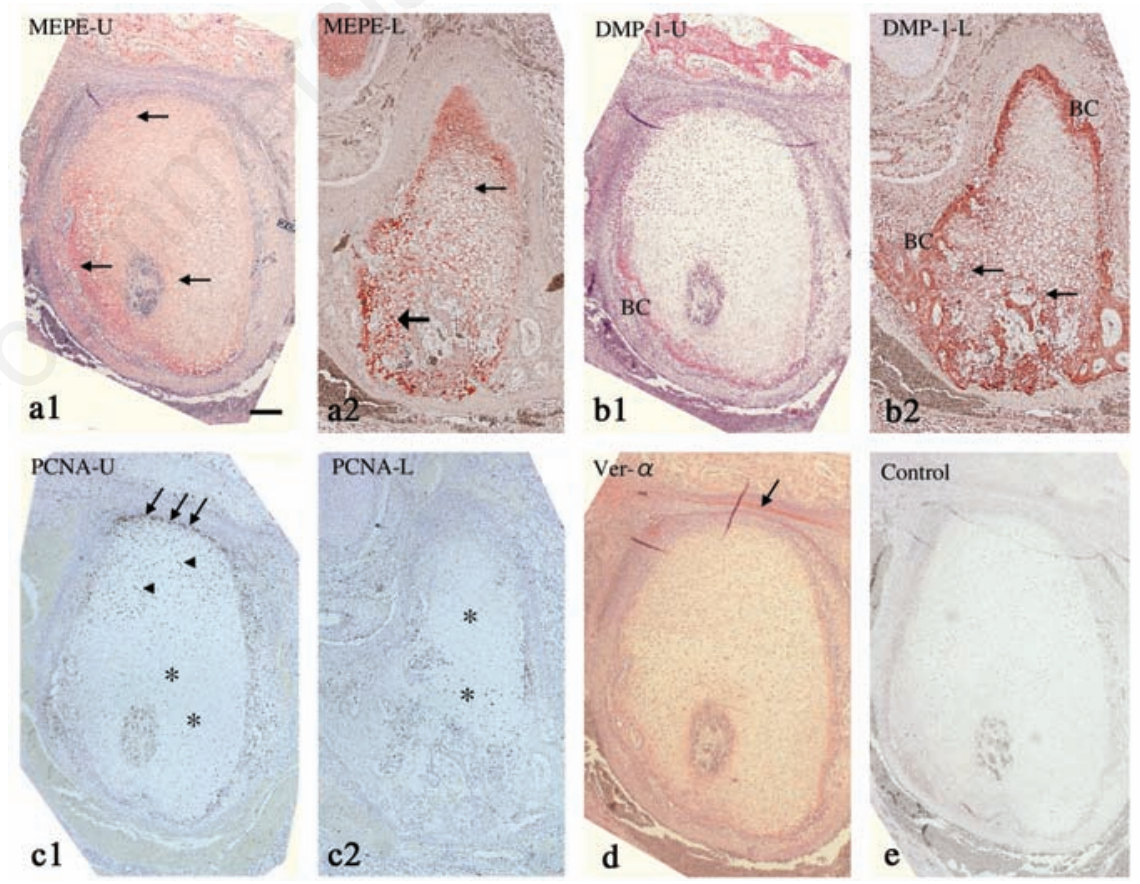

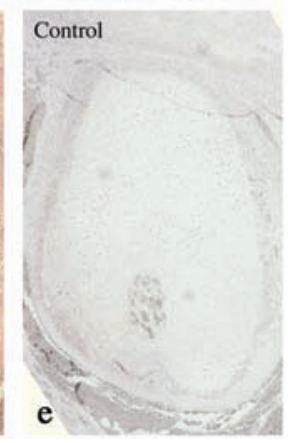

Figure 4. a1,b1,c1, a2,b2,c2) are adjacent sections to Figure 3 a1 and a2, respectively, and immunostaining for MEPE (a1,a2), DMP-1 (b1,b2), and PCNA (c1,c2). d,e) are also adjacent sections to Figure 3a, and immunostaining for versican GAG- $\alpha$ domain (d), and a negative control reacted with normal rabbit IgG (e). Condylar cartilage showed immunoreactivity for MEPE moderately in the upper layers and intensely in the deep layer of the hypertrophic cell layer $(\mathrm{a} 1, \mathrm{a} 2)$, and for DMP-1 weakly in the deep layer of the hypertrophic cell layer (b2). Bone collar showed intense DMP-1 immunoreactivity (b2). PCNA immunoreactivity was mainly evident in the mesenchymal layer (c1). Versican GAG - $\alpha$ domain was evident in joint cavities (d). BC, bone collar. Scale bars: $200 \mu \mathrm{m}$. 
that this molecule is present on the outer annulus fibrosus, in marginal tissues around the vertebral body, and in developing intervertebral discs. Their results were comparable to ours in that tissues around cartilage have type I collagen immunoreactivity.

In condylar cartilage, aggrecan immunoreactivity was evident throughout the cartilaginous matrix. Type II collagen immunoreactivity was also evident throughout the cartilaginous matrix, but it was not as extensive as that in Meckel's cartilage. In contrast, type I collagen immunoreactivity was more evident than type II collagen immunoreactivity in the cartilaginous matrix, especially in the articular layer, the chondroblastic layer and in the deep layer of the hypertrophic cell layer; these findings indicate that condylar cartilage has a fibrocartilaginous nature. This nature is different from that of Meckel's cartilage and cranial base cartilage, but comparable to condylar cartilage in rats and mice..$^{10-13}$ Mandibular condylar cartilage in mice arises from an alkaline phosphatase-positive periosteum-like tissue, not from original mesenchymal tissue, ${ }^{5,6}$ and hence, this fibrocartilaginous nature reflects a periosteum origin of mouse condylar cartilage. This distinction is a major difference between primary and secondary cartilage, and our findings indicated that the distinction is applicable to human fetal craniofacial cartilage. Type $\mathrm{X}$ collagen immunoreactivity was evident from the chondrocyte layer to the hypertrophic cell layer, indicating that the condylar cartilage in this stage comprises a relatively thick layer when compared to cranial base cartilage and has characteristics of hypertrophic chondrocytes. In mice, progenitor cells of condylar cartilage rapidly differentiate into hypertrophic chondrocytes, and the hypertrophic cell layer extends in length remarkably in a few days. ${ }^{5}$ Thus, mouse fetal condylar cartilage has a relatively thick hypertrophic cell layer, and this attribute is another structural feature of condylar (secondary) cartilage that distinguishes condylar cartilage from primary cartilage. Taken together, these findings indicated that the concept of secondary cartilage is applicable to the condylar cartilage of the human fetus.

In cranial base cartilage, we accepted the general classification of zones in long bone cartilage, but PCNA immunoreactivity was not intense in the proliferation zone. Thus, fetal cranial base cartilage seems not to harbor substantial proliferation activity; however, we did not perform PCNA immunohistochemistry in fetal long bone cartilage. In condylar cartilage, PCNA immunoreactivity was mainly detected in the mesenchymal layer, and these results are comparable to those from a bromodeoxyuridine (BrDU)-labeling study of growing rats, ${ }^{45}$ but not to our findings from the cranial base cartilage. Thus, human fetal condylar cartilage has a pattern of proliferation that is similar to that of rodent growing condylar cartilage.

\section{Expression of versican, MEPE, and DMP-1 in craniofacial cartilage and bone}

Versican (G1 domain) immunoreactivity was only evident in the perichondrium and future joint cavities in the present study. Versican is a fetal proteoglycan found in various tissues; ${ }^{14-17}$ therefore, versican immunoreactivity should have been detected widely and in many of the other tissues examined. The lack of versican immunoreactivity may be due to processed form of this molecule, since versican has four splice-variants (V0, V1, V2, V3). ${ }^{14}$ However, this possibility is low, since versican GAG- $\alpha$ domain immunoreactivity showed similar expression patterns. We speculate that decalcification during tissue preparation affected staining intensity, since human fetal brain in undecalcified sections show good immunoreactivity for this antibody (data not shown). Thus some tissues such as the perichondrium and upper joint cavity may be versican rich despite the lack of versican immunoreactivity seen in this study.

DMP-1 is in the SIBLING protein family and was initially reported to be an odontoblast-specific molecule, ${ }^{18}$ but was later found to be strongly expressed in osteocytes ${ }^{19-21}$ and weakly expressed in other mineralized tissues such as cartilage, enamel, and dental pulp. ${ }^{22,23} \mathrm{~A}$ study of DMP-1-deficient mice indicates that this molecule is linked to amelogenesis, cement genesis, dentinogenesis, chondrogenesis, and osteogenesis during development. ${ }^{22}$ Interestingly, mechanical loading may induce DMP-1 expression in osteocytes. ${ }^{21}$ In the present study, DMP-1 immunoreactivity was evident in bone matrix (bone lacunae walls and canaliculi) as well as in the lower layer of the hypertrophic cell zone both in cranial base car-
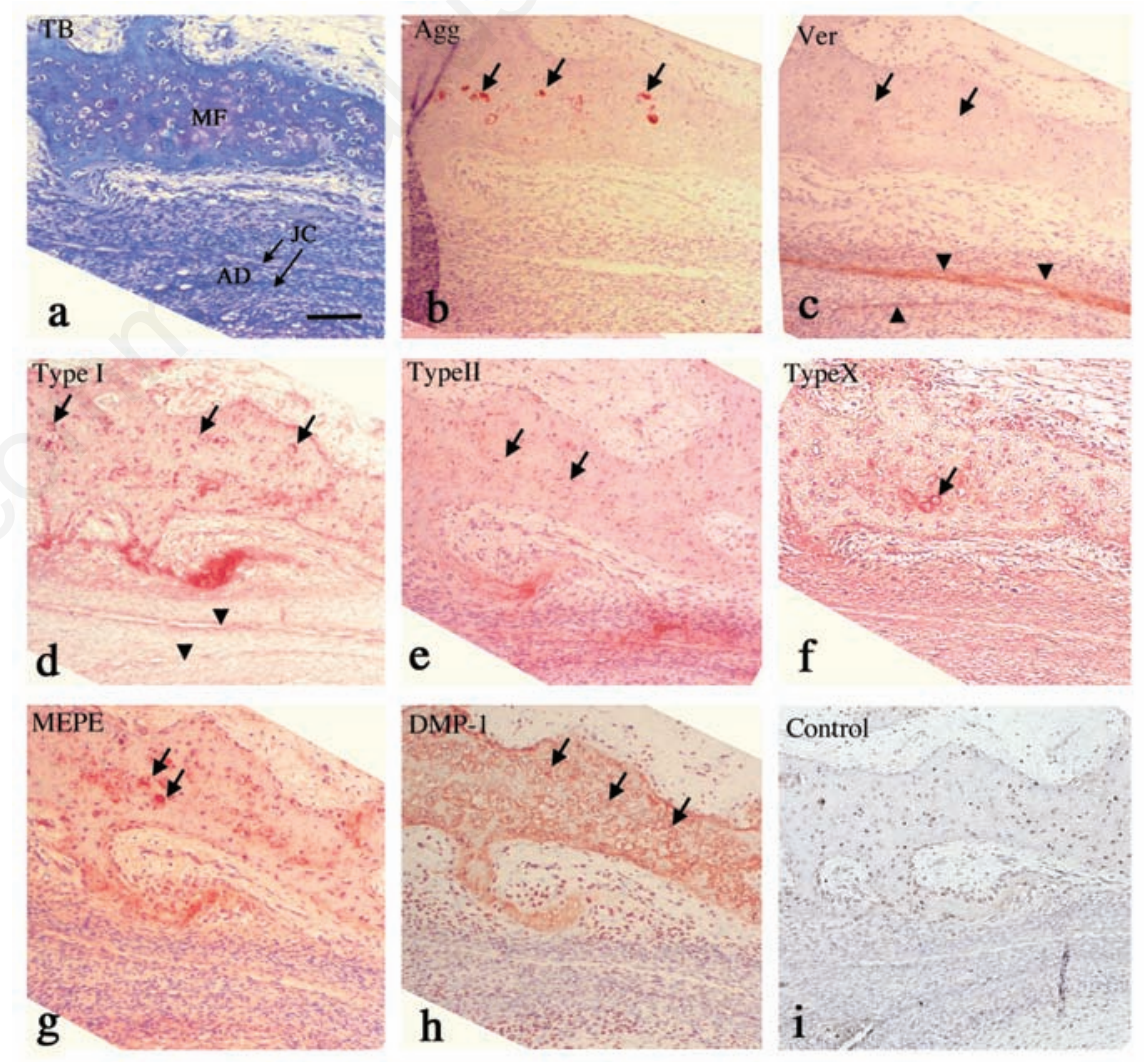

Figure 5. Mandibular fossa and anlagen of articular cavity at 16 weeks of gestation. a) is higher magnification of rectangular area of Figure $3 a$, and $b-i$ are adjacent sections to a. Toluidine blue staining (a) and immunostaining for aggrecan (b), versican (c), collagen types I (d), II (e), and X (f), MEPE (g), DMP-1 (h), and a negative control reacted with normal rabbit IgG (i). Most of bone lacunae showed immunoreactivity for type I collagen (d) and DMP-1 (h). A few lacunae showed immunoreactivity for aggrecan (b), type X collagen (f), and MEPE (g). Anlagen of future articular joint cavity showed immunoreactivity for versican intensely (c) and for type I collagen slightly (d). MF, mandibular fossa; $\mathrm{AD}$, articular disc; JC, joint cavity. Scale bars: $100 \mu \mathrm{m}$. 
tilage and condylar cartilage. This staining pattern was comparable to that seen in mice, and hence, the proposed function of DMP-1 in mice may be relevant to its function in human fetuses. MEPE is another member of the SIBLING family of proteins and is also more strongly expressed in osteocytes than in osteoblasts. ${ }^{24-26}$ This molecule is also expressed in response to mechanical loading. ${ }^{26}$ In the present study, a small number of bone lacunae in the mandibular bone showed MEPE immunoreactivity, and this result was comparable to those in mice. ${ }^{24,25}$ However, strong MEPE immunoreactivity was evident in the matrix of Meckel's cartilage, cranial base cartilage, and condylar cartilage in human fetuses. Reportedly, MEPE is expressed in odontoblasts and dental pulp cells, ${ }^{27-30}$ but
MEPE expression in cartilage has only been seen in late-stage hypertrophic chondrocytes of $c$-Src-deficient mice ${ }^{31}$ and during regeneration of the fracture callus in mice. ${ }^{32}$ Since the hypertrophic chondrocytes in $c$-Src-deficient mice have osteocytic characteristics, our findings are the first documentation of MEPE expression in normal cartilage matrix. MEPE immunoreactivity was also evident in Meckel's cartilage of fetal mice and fetal rats; therefore, we can regard this molecule as a component of the cartilage matrix in mammalian fetuses. Previous studies indicated that MEPE is involved in mineralization in bone and dentin..$^{24,28,32}$ In the present study, enhanced MEPE immunoreactivity was evident in the cartilaginous core of the trabecular bone in the
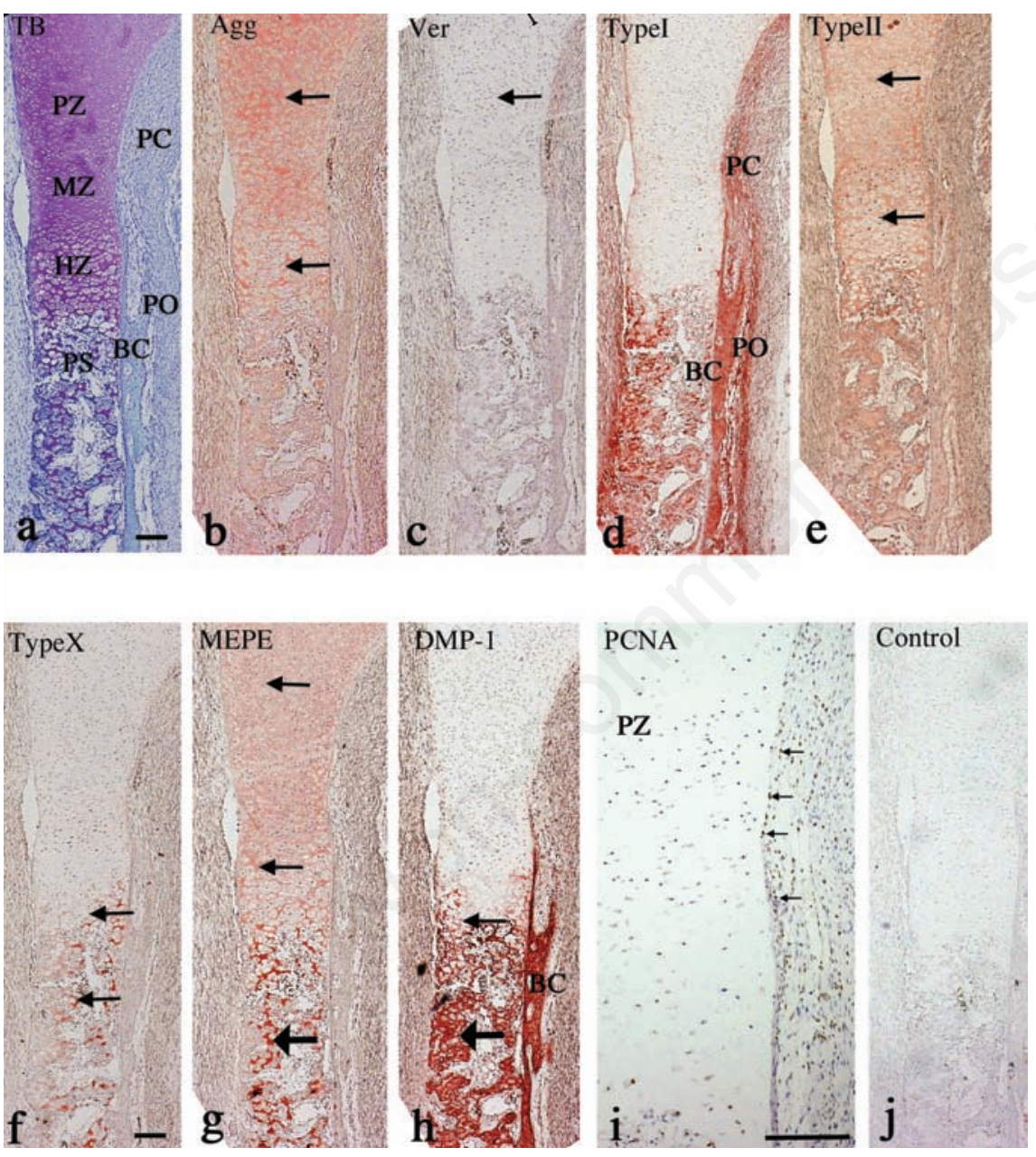

Figure 6. Cranial base cartilage at 16 weeks of gestation. Toluidine blue staining (a) and immunostaining for aggrecan (b), versican (c), collagen types I (d), II (e), and X (f), MEPE (g), DMP-1 (h), PCNA (i), and a negative control reacted with normal rabbit IgG (j). Cranial base cartilage showed immunoreactivity for aggrecan (b), type II collagen (e), and MEPE (g) throughout entire cartilage matrix, and for type $X$ collagen (f) and DMP1 (h) exclusively in the hypertrophic cell zone. Note enhanced MEPE immunoreactivity in the cartilaginous core in the primary spogiosa (large arrow in g). Bone collar showed immunoreactivity for type I collagen (d) and DMP-1 (h). Periosteum and perichondrium showed immunoreactivity for type I collagen (d). PCNA immunoreactivity was mainly detected in the perichondrium (i). BC, bone collar; PO, periosteum; PC, perichondrium; PZ, proliferative zone; MZ, maturation zone; HZ, hypertrophic cell zone; PS, primary spongiosa. Scale bars: $200 \mu \mathrm{m}$.

primary spongiosa. Thus we speculate that MEPE is also involved in mineralization of cartilage, but its primary function in cartilage remains unknown.

\section{Structural features of mandibular (glenoid) fossa}

In the ossifying mandibular fossa, some lacunae showed aggrecan, type $\mathrm{X}$ collagen, and MEPE immunoreactivity, indicating that the ossifying mandibular fossa contained some chondrocyte lacunae. Reportedly, cartilaginous tissue is present in the mandibular fossa or the continuous articular eminence of rats, ${ }^{46,47}$ young monkeys. ${ }^{48}$ Only a few studies have investigated whether this type of cartilage exists in the mandibular fossa of the human fetus. ${ }^{39}$ Many lacunae in the mandibular fossa showed immunoreactivity for type I collagen and DMP-1. Thus, aggrecan-positive lacunae were surrounded by osteocyte lacunae. Tissues with these characteristics are often classified as chondroid bone. ${ }^{3,46}$ According to this criterion, the ossifying mandibular fossa seen in the present study can be classified as chondroid bone. Mizoguchi et al. ${ }^{46}$ described co-localization of collagen types I, II, X, and osteocalcin in the chondroid bone of rat mandibular fossa and insisted that chondroid bone has phenotypic expression associated with hypertrophic chondrocytes and osteocytes. The present results were similar to their results. When compared to bone, cartilage has an advantage for skeletal growth because cartilage can undergo both appositional and interstitional growth. ${ }^{2}$ Thus, emergence of cartilaginous tissue (chondroid bone) in this region may facilitate the development of the mandibular fossa.

\section{References}

1. Nanci A. Temporomandibular joint. In: Ten Cate's oral histology, development, structure, and function. 7th ed. St Louis: Mosby; 2008, p. 358-78.

2. Proffit WR. Concept of Growth and Development. In: WR Proffit, HW Fields, DH Sarver (eds.) Contemporary orthodontics, 5th ed. St Louis: Mosby; 2013, p. 20 65 .

3. Beresford WA. Chondroid bone, secondary cartilage and metaplasia. Baltimore: Urban \& Schwarzenberg; 1981, p.23-47.

4. Vinkka H. Secondary cartilages in the facial skeleton of the rat. Proc Finn Dent Soc 1982;78(Suppl.7):1-137.

5. Shibata S, Fukada K, Suzuki S, Yamashita Y. Immunohistochemistry of collagen types II and X, and enzyme-histochemistry of alkaline phosphatase in the developing 
condylar cartilage of the fetal mouse mandible. J Anat 1997;191:561-70.

6. Shibata S, Yokohama-Tamaki T. An in situ hybridization study of Runx2, Osterix, and Sox9 in the anlagen of mouse mandibular condylar cartilage in the early stages of embryogenesis. J Anat 2008;213:274-83.

7. Hall BK. Bones and cartilage: developmental and evolutionary skeletal biology. San Diego, London: Elsevier Academic press; 2005, p. 149-65.

8. Durkin JF, Heerly JD, Irving JT. The cartilage of mandibular condyle. Oral Sci Rev 1973;2:29-99.

9. Shibata S, Fukuoka H, Sato R, Abe T, Suzuki Y. An in situ hybridization study of the IGF system in developing condylar cartilage of the fetal mouse mandible. Eur $\mathrm{J}$ Histochem 2012;56:e23.

10. Mizoguchi I, Nakamura M, Takahashi I, Kagayama M, Mitani H. An immunohistochemical study of localization of type I and type II collagens in mandibular condylar cartilage compared with tibial growth plate. Histochemistry 1990;93:593-9.

11. Ishii M, Suda N, Tengan T, Suzuki S, Kuroda T. Immunohistochemical findings type I and type II collagen in prenatal mouse mandibular condylar cartilage compared with the tibial anlage. Arch Oral Biol 1998;43:545-50.

12. Delatte M, Von den Hoff JW, van Rheden REM, Kuijpers-Jagtman AM. Primary and secondary cartilages of the neonatal rat: femoral head and the mandibular condyle. Eur J Oral Sci 2004;112:156-62.

13. Ohashi N, Ejiri S, Hanada, Ozawa H. Changes in type I, II, X collagen immunoreactivity of the mandibular condylar cartilage in a naturally aging rat model. J Bone Miner Metab 1997;15:77-83.

14. Shinomura T, Nishida Y, Ito K, Kimata K. cDNA cloning of PG-M, a large chondroitin sulfate proteoglycan expresses during chondrogenesis in chick limb buds. Alternative spliced multiforms of PG-M and their relationships to versican. J Biol Chem 1993;268:17640-7.

15. Shibata S, Fukada K, Suzuki, S, Ogawa T, Yamashita Y. Histochemical localization of versican, aggrecan and hyaluronan in the developing condylar cartilage of the fetal rat mandible. J Anat 2001;198:129-35.

16. Shibata S, Kaneko, S, Yanagishita M, Yamashita Y. Histochemical localization of hyaluronan and versican in the rat molar dental pulp. Arch Oral Biol 2000;44:563-8.

17. Murakami T, Ohtsuka A. Perisynaptic barrier of proteoglycans in the mature brain and spinal cord. Arch Histol Cytol 2003; 66:195-207.

18. George A, Sabsay B, Simonian PA, Veis A. Characterization of a novel dentin matrix acidic phosphoprotein. Implications for induction of biomineralization. J Biol Chem 1993;268:12624-30.

19. Toyosawa S, Shintani S, Fujiwara T, Ooshima T, Sato A, Ijuhin N, et al. Dentin matrix protein 1 is predominantly expressed in chicken and rat osteocytes but not in osteoblasts. J Bone Miner Res 2001;16:2017-26.

20. Baba 0, Qin C, Brunn JC, Wygant JN, McIntyre BW, Butler WT. Colocalization of dentin matrix protein 1 and dentin sialoprotein at late stages of rat molar development. Matrix Biol 2004;23:371-9.

21. Gluhak-Heinrich J, Ye L, Bonewald LF, Feng JQ, MacDougall M, Harris SE, et al. Mechanical loading stimulates dentin matrix protein 1 (DMP1) expression in osteocytes in vivo. $\mathrm{J}$ Bone Miner Res 2003;18:807-17.

22. Feng JQ, Zhang J, Dallas SL, Lu Y, Chen S, Tan X, et al. Dentin matrix protein 1, a target molecule for cbfal in bone, is a unique bone marker gene. J Bone Miner Res 2002;17:1822-31.

23. Feng JQ, Huang H, Lu Y, Ye L, Xie Y, Tsutsui TW, et al. The dentin matrix protein 1 (Dmpl) is specifically expressed in mineralized, but not soft, tissues during development. J Dent Res 2003;82:776-80.

24. Gowen LC, Peterson DN, Mansolf, AL, Qi H, Stock JL, Tkalcevic GT, et al. Targeted disruption of the osteoblast/osteocyte factore 45 gene (OF45) result in increased bone formation and bone mass. $\mathrm{J}$ Biol Chem 2003;278:1998-2007.

25. Igarashi M, Kamiya N, Ito K, Takagi M. In situ localization and in vitro expression of osteoblasts/osteocyte factor 45 mRNA during bone cell differentiation. Histochem $\mathrm{J}$ 2002;34:255-63.

26. Gluhak-Heinrich J, Pavlin D, Yang W, MacDougall M, Harris SE. MEPE expression in osteocytes during orthodontic tooth movement. Arch Oral Biol 2007; 52:684-90.

27. Chen S, Chen L, Jahangiri A, Chen, B, Wu $\mathrm{Y}$, Chuang HH, et al. Expression and processing of small integrin-binding ligand $\mathrm{N}$ linked glycoproteins in mouse odontoblastic cells. Arch Oral Biol 2008;53:879-89.

28. Wang HG, Kawashima N, Iwata T, Xu J, Takahashi S, Sugiyama T, et al. MEPE activated by Furin promotes pulpal cell adhesion. J Dent Res 2011;90:529-34.

29. Hou C, Liu ZV, Tang KL, Wang MG, Sun J, Wang J, et al. Developmental changes and regional localization of Dspp, Mepe, Mimecan and Versican in postnatal developing mouse teeth. J Mol Histol 2012;43:916.

30. Liu H, Li W, Shi S, Habelitz S, Gao C, DenBesten P. MEPE is down regulated as dental pulp stem cells differentiate. Arch Oral Biol 2005;50:923-8.

31. Shibata S, Baba 0, Oda T, YokohamaTamaki T, Qin C, Butler WT, et al. An immunohistochemical and ultrastructural study of the pericellular matrix of uneroded hypertrophic chondrocytes in the mandibular condyle of aged c-src-deficient mice. Arch Oral Biol 2008;53:20-30.

32. Lu C, Huang S, Miclau T, Helms JA, Colnot C. Mepe is expressed during skeletal development and regeneration. Histochem Cell Biol 2004;121:493-9.

33. Suda N, Shibata S, Yamazaki K, Kuroda T, Senior PV, Beck F, et al. Parathyroid hormone -related protein (PTHrP) regulates proliferation of condylar hypertrophic chondrocytes. J Bone Miner Res 1999;14: 1838-47.

34. Smith SM, Shu C, Melrose J. Comparative immunolocalisation of perlecan with collagen II and aggrecan in human fetal, newborn and adult ovine joint tissues demonstrates perlecan as an early developmental chondrogenic markers. Histochem Cell Biol 2010;134:251-63.

35. Smith SM, Whitelock JM, Iozzo RV, Littlel CB, Melrose J. Topographical variation in the distribution of versican, aggrecan, and perlecan in the fetal human spine reflects their diverse functional roles in spinal development. Histochem Cell Biol 2009; 132:491-503.

36. Mérida-Velasco JR, Rodríguez-Vázquez JF, Mérida-Velasco JA, Sánchez-Montesinos I, Espín-Ferra J, Jiménez-Collado J. Development of the human tempromandibular Joint. Anat Rec 1999;255:20-33.

37. Mérida-Velasco JR, Rodríguez-Vázquez JF, De la Cuadra-Blanco C, Campos-López R, M, Sánchez-Montesinos I, Mérida-Velasco JA. Development of the mandibular condylar cartilage in human specimens of 10-15 weeks' gestation. J Anat 2009;214:56-64.

38. Carini F, Scardina GA, Caradonna C, Messina P, Valenza V. Human temporomandibular joint morphogenesis. Ital $\mathrm{J}$ Anat Embryol 2007;112:267-75.

39. Martinez G, Caltabiano C, Leonardi R, Caltabiano M. [Histomorphology of secondary cartilage in human fetal mandible]. [Article in Italian]. Minerva Stomatol 1997:46:39-43.

40. Radlanski RJ, Lieck S, Bontschev NE. Development of the human temporomandibular joint. Computer aided 3Dreconstructions. Eur J Oral Sci 1999;107: 25-34.

41. Shibata S, Sato R, Murakami G, Fukuoka, H, Rodríguez-Vázquez, JF. Origin of mandibular condylar cartilage in mice, rats and humans: periosteum or separate blastema? J Oral Biosci 2013;55:208-16. 
42. de Moraes LOC, Lodi FR, Gomes, TS, Marques, SR, Oshima CTF, Lancelotti CLP, et al. Immunohistochemical expression of types I and III collagen antibodies in the temporomandibular joint disc of human fetuses. Eur J Histochem 2011;55:e24.

43. Kiga N, Tojyo I, Matsumoto T, Hiraishi Y, Shinoyama Y, Fujita S. Expression of lumican related to CD34 and VEGF in the articular disc of human temporomandibular joint. Eur J Histochem 2010;54:e34.

44. Kiga N, Tojyo I, Matsumoto T, Hiraishi Y, Shinohara Y, Makino S, et al. Expression of lumican and fibromodulin following interleukin-1 beta stimulation of disc cells of the human temporomandibular joint. Eur J Histochem 2011;55:e11.

45. Luder HU, Leblond CP, von der Mark K. Cellular stages in cartilage formation as revealed by morphometry, radioautography and type II collagen immunostaining of the mandibular condyle from weanling rats. Am J Anat 1988;182:197-214.

46. Mizoguchi I, Takahashi I, Sasano Y, Kagayama M, Kuboki Y, Mitani $\mathrm{H}$. Localization of type I, II and X collagen and osteocalcinin intramembranous, endochondral and chodroid bone of rats. Atat Embryol 1997;196:291-7.

47. Owtad P, Potres Z, Schen G, Petocz P, Darendelier MA. A histochemical study on condylar cartilage and glenoid fossa during mandibular advancement. Angle Orthod. 2011;81:270-6.

48. Furseth Klinge R. The structure of the fibrous tissue on the articular surface of the temporal bone in the monkey (Macaca Mulatta). Micron 2002;32:551-7. 